\title{
Hereditary Afibrinogenemia: Rare Bleeding Disorder Presenting with Spontaneous Extradural Hematoma - A Brief Review
}

\author{
Guru Dutta Satyarthee ${ }^{1}$ Ashok K. Mahapatra ${ }^{2}$ \\ ${ }^{1}$ Department of Neurosurgery, All India Institute of Medical Science, \\ New Delhi, India \\ 2 Department of Neurosurgery, All India Institute of Medical Science, \\ Bhuvneshwar, India
}

\begin{abstract}
Address for correspondence Guru Dutta Satyarthee, MCh, Department of Neurosurgery, Room no. 714, Neurosciences Centre, All India Institute of Medical Science, Ansari Nagar East, Delhi 110029, India (e-mail: duttaguru2002@yahoo.com).
\end{abstract}

Indian J Neurosurg 2016;5:22-24.

\begin{abstract}
Keywords

- afibrinogenemia

- fresh frozen plasma

- rare bleeding disorders

- extradural hematoma

Afibrinogenemia is considered as rare hereditary bleeding disorder with autosomal recessive genetic transmission, caused by mutations of any one out of the three genes located on chromosome 4, responsible for coding of three polypeptide chains, constituents of fibrinogen. Clinical manifestation include spontaneous bleeding, bleeding following trivial trauma, and excessive profuse bleeding during major trauma constitute commoner manifestations. Replacement therapy remains main treatment of bleeding episodes using plasma-derived fibrinogen concentrate. However, fresh frozen plasma and cryoprecipitate, recombinant fibrinogens are considered as alternative treatment options. The authors reports a 2-year-old girl, diagnosed as case of hypofibrinogenemia since birth and was under regular supervision of pediatric hematologist, getting fresh frozen plasma replacement therapy every third weeks, developed extradural hematoma and was managed surgically with good outcome.
\end{abstract}

\section{Introduction}

Afibrinogenemia is an extremely rarer hereditary bleeding disorder characterized by spectrum of bleeding manifestations. Fibrinogen constitutes an essential clotting factor and plays important role in clot formation. Fibrinogen converts into fibrin by the action of thrombin. ${ }^{1-4}$ Further fibrinogen also plays critical role in primary hemostasis as it promotes to aggregation of platelet by binding to glycoprotein IIb/IIIa present on the surface of activated platelets. ${ }^{5}$ Author reports a 2-year-old girl's case known case of afibrinogenemia, diagnosed at age of 4 days of neonatal life as she had excessive bleeding from umbilical stump. Since then she was under regular follow-up at hematology department of our institute and received prophylactic fresh frozen plasma transfusion every third week. She presented with extradural hematoma, was managed with evacuation of hematoma surgically, after receiving fresh frozen plasma on arrival in emergency department and during perioperative period.

received

June 18, 2015

accepted

November 18, 2015

published online

February 25, 2016

\section{Case Illustration}

This female baby was the first issue born to parents with nonconsanguineous marriage. The prenatal period was uneventful. The baby was born at full term through normal vaginal delivery. On fourth days after the birth, the mother noticed excessive bleeding from umbilical stump. She was admitted in the neonatal unit and pediatrician consulted hematologist. She was diagnosed as case of afibrinogenemia and further advised for regular transfusion of fresh frozen plasma, cryoprecipitate, or recombinant fibrinogen replacement therapy, but parent opted for fresh frozen plasma because of economic constrain and accordingly she was regularly receiving fresh frozen plasma replacement therapy since neonatal period. She was doing well, suddenly developed episodes of excessive inconsolable cries associated with multiple vomiting, though there was no definite history of trauma, or febrile illness or exanthematous rashes, and 2 hours later she was brought
License terms of India 


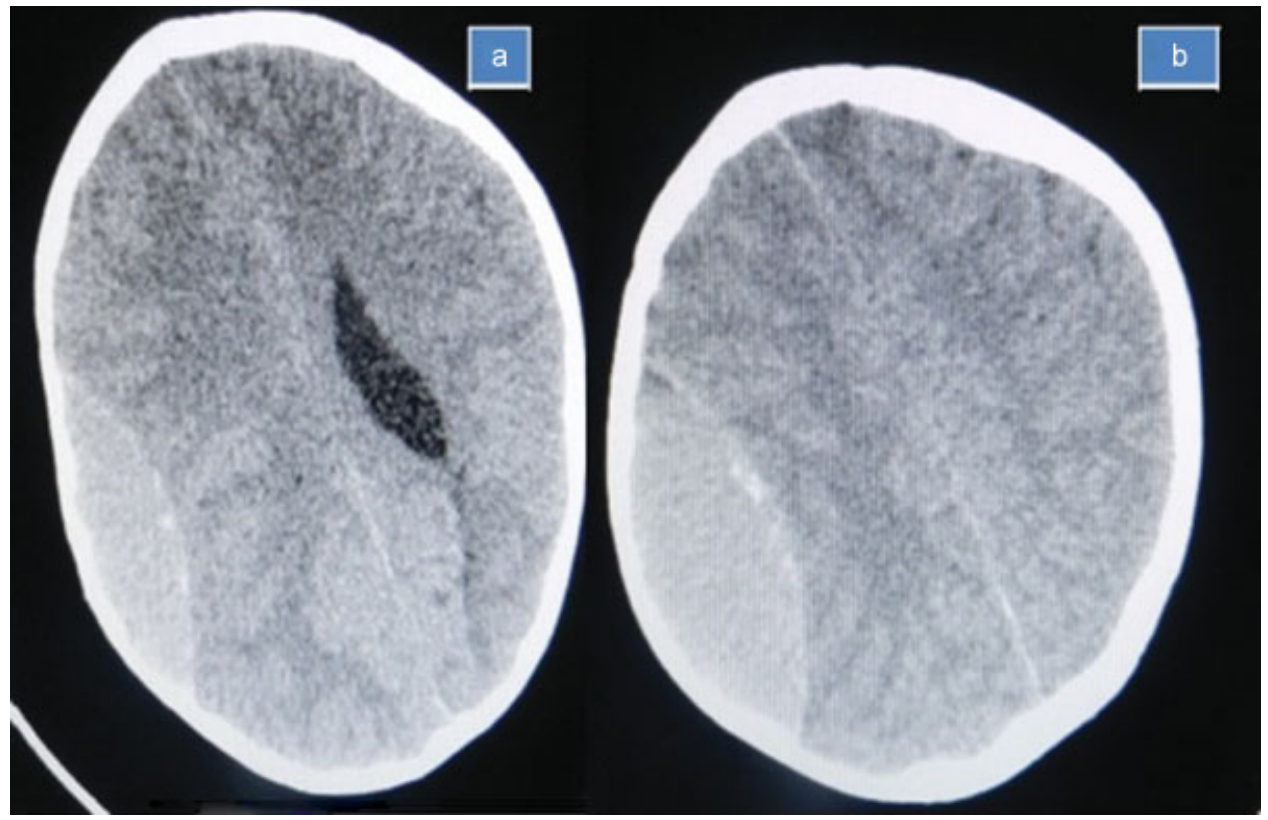

Fig. 1 NCCT of the head showing right parietal extradural hematoma causing mass effect.

to emergency services. On examination, pulse rate was 88 beats/min, head circumference was $52 \mathrm{~cm}$; pupillary asymmetry and rest of examination was essentially within normal limits. She was subjected immediately to noncontrast computed tomographic (NCCT) scan of the brain (-Fig. 1) that showed a large hyperdense extra-axial mass lesion in the right temporoparietal region from the atrium causing pressure effect on ipsilateral ventricle. Immediately hematologist consultation sought and received two pediatric units of fresh frozen plasma in the emergency department and planned for emergency evacuation of extradural hematoma.
The patient was given general anesthesia and positioned supine, and right-sided parietal osteoplastic craniotomy was performed using Midas Rex drill. On bone removal, clotted blood that was causing pressure effect was evacuated. Hemostasis was achieved and wound closed in layer after putting extradural drain. She tolerated surgery well and postoperative period was uneventful. NCCT scan of the head in the postoperative phase showed complete removal of extradural hematoma (-Fig. 2). The baby was discharged on postoperative day 3 and she was taking oral feeds with no further episodes of vomiting. She was doing well at the last follow-up at 6 months.

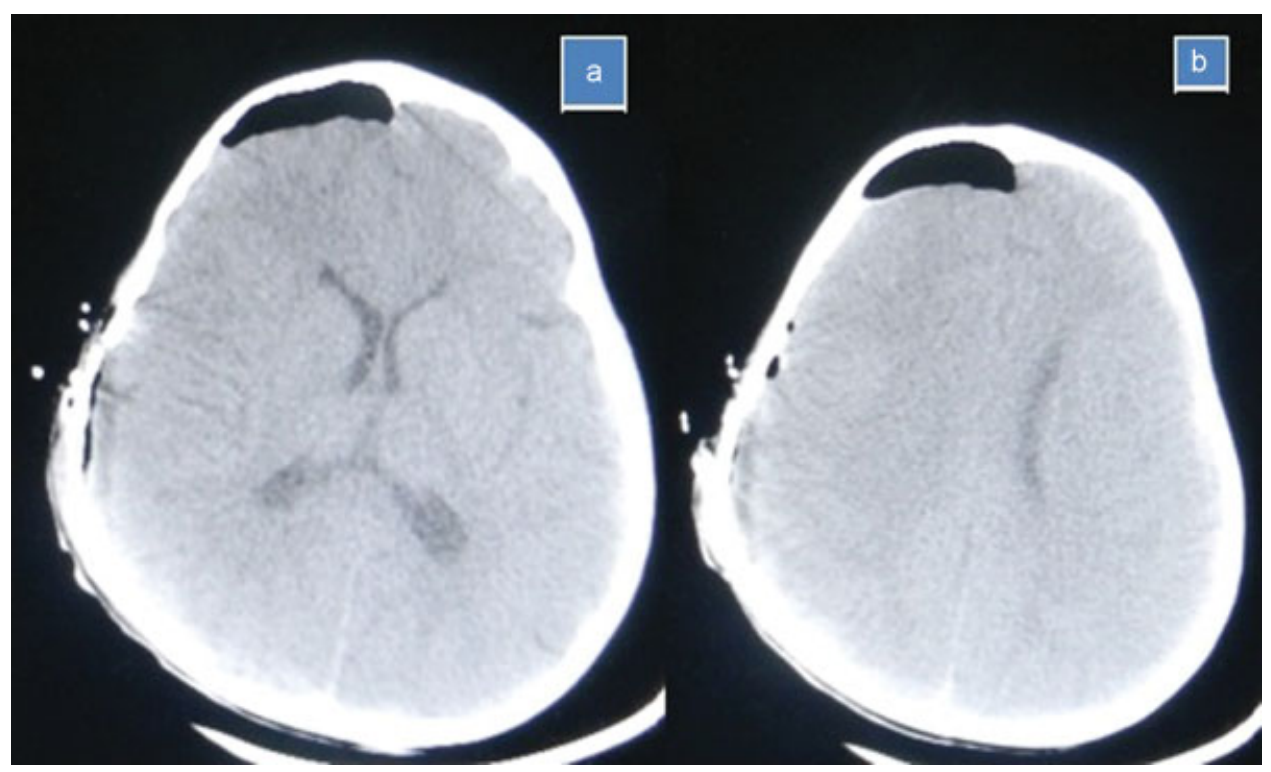

Fig. 2 Postoperative NCCT of the head showing evacuation parietal extradural hematoma with relief in mass effect and reversal of falcine herniation. 


\section{Discussion}

Afibrinogenemia is a rare hereditary bleeding disorder having autosomal recessive genetic transmission and prevalence of $1: 1,000,000$ live birth. ${ }^{2}$ The fibrinogen has three polypeptide chains, that is, $A \alpha, B \beta$, and $\gamma$, controlled by corresponding three genes FGA, FGB, and FGG, located on chromosome 4. Mutation of any one of three genes may affect synthesis or release of fibrinogen during formation, assembly, stability, or secretion phases of fibrinogen. ${ }^{4}$ The Patient with afibrinogenemia shows prolonged prothrombin time, thrombin, and activated partial thromboplastin time, undetectable functional fibrinogen and absence or immunoreactive fibrinogen with deranged platelet adhesion and adenosine diphosphate (ADP)-induced platelet aggregation but normal thrombin- and collagenstimulated platelet aggregation. $^{5}$

The most common symptoms are umbilical stump bleeding and hemorrhage from mucosal surfaces, menorrhagia, epistaxis, and bleeding inside oral cavity. $3,5,6$ Bleeding in the joint space and excessive bleeding during surgical procedures are other principal manifestations, whereas spontaneous intracranial bleeding is extremely rare, ${ }^{6}$ although it can develop following minor trauma. ${ }^{2,3,6,7}$

Management of afibrinogenemia requires constant vigilance and anticipation; however, during active bleeding episodes, replacement therapy remains main modalities and includes intravenous administrations of plasma-derived fibrinogen concentrate, cryoprecipitate, and fresh frozen plasma or recombinant synthetic fibrinogen. ${ }^{5}$ As half-life of fibrinogen is approximately 4 days and can be administered daily or alternate day, need is based on indication, response, and result of hematologic laboratory investigation. For spontaneous bleeding, recommended target levels of fibrinogen should be more than $1 \mathrm{~g} / \mathrm{L}$ till securing complete hemostasis and during maintenance phase, target till healing is greater than $0.5 \mathrm{~g} / \mathrm{L}^{5-7}$

Fibrinogen concentrate is replacement therapy of choice, its advantages is virally inactivated. It needs relatively smaller infusion volume of transfusion and associated reduced allergic reaction. ${ }^{2}$ However, fresh frozen plasma and cryoprecipitate are another alternative replacement agent, and it can be administered in emergencies where fibrinogen concentrate availability is uncertain. However, our patient received fresh frozen plasma during peri- and postoperative periods of extradural hematoma evacuation as she was regularly receiving during follow-up under hematology physician since neonatal period. Prior to surgical intervention, fibrinogen levels should be built up to at least $1 \mathrm{~g} / \mathrm{L}$ and maintained at the same level till completion of surgery; however, major surgical procedures in these cases, as a precautionary measure should be performed in a well-equipped centers possessing wellestablished and functioning blood bank. ${ }^{8,9}$
Indications of prophylactic infusion of fibrinogen concentrate or cryoprecipitate are following lifethreatening bleeding episodes. Prophylactic therapy includes weekly infusions in most patients, but transfusion every 2 weeks or once a month has also been used. ${ }^{6}$ According to recommendation of current guidelines, regarding consideration for secondary prophylactic treatment where risk of life-threatening bleeding and with a target fibrinogen level should be $0.5 \mathrm{~g} / \mathrm{L} .{ }^{9}$ Usually, primary prophylactic infusion is not advocated as associated risk of transmission of infectious diseases, allergic reactions, and rare thrombotic complications are very high. ${ }^{5,8,9}$

\section{Conclusion}

Constant vigilance is required for detecting early intracranial hemorrhage, and replacement therapy should be transfused at earliest opportunity to correct fibrinogen deficiency state and should be continued in the perioperative period. Pediatrician as well as neurosurgeon should be aware of existence of such rare bleeding disorder while managing such cases in the emergency services.

\section{References}

1 Acharya SS, Coughlin A, Dimichele DM; North American Rare Bleeding Disorder Study Group. Rare Bleeding Disorder Registry: deficiencies of factors II, V, VII, X, XIII, fibrinogen and dysfibrinogenemias. J Thromb Haemost 2004;2(2): 248-256

2 Mannucci PM, Duga S, Peyvandi F. Recessively inherited coagulation disorders. Blood 2004;104(5):1243-1252

3 Lak M, Keihani M, Elahi F, Peyvandi F, Mannucci PM. Bleeding and thrombosis in 55 patients with inherited afibrinogenaemia. $\mathrm{Br} \mathrm{J}$ Haematol 1999;107(1):204-206

4 Asselta R, Duga S, Tenchini ML. The molecular basis of quantitative fibrinogen disorders. J Thromb Haemost 2006; 4(10):2115-2129

5 Acharya SS, Dimichele DM. Rare inherited disorders of fibrinogen. Haemophilia 2008;14(6):1151-1158

6 Castaman G. Prophylaxis of bleeding episodes and surgical interventions in patients with rare inherited coagulation disorders. Blood Transfus 2008;6(Suppl 2):s39-s44

7 Dupuy E, Soria C, Molho P, et al. Embolized ischemic lesions of toes in an afibrinogenemic patient: possible relevance to in vivo circulating thrombin. Thromb Res 2001;102(3): 211-219

8 Keeling D, Tait C, Makris M. Guideline on the selection and use of therapeutic products to treat haemophilia and other hereditary bleeding disorders. A United Kingdom Haemophilia Center Doctors' Organisation (UKHCDO) guideline approved by the British Committee for Standards in Haematology. Haemophilia 2008;14(4):671-684

9 Bolton-Maggs PH, Perry DJ, Chalmers EA, et al. The rare coagulation disorders-review with guidelines for management from the United Kingdom Haemophilia Centre Doctors' Organisation. Haemophilia 2004;10(5):593-628 